\begin{tabular}{|c|c|c|c|c|c|c|}
\hline \multirow{4}{*}{ Impact Factor: } & ISRA (India) & $=3.117$ & SIS (USA) & $=0.912$ & ICV (Poland) & $=6.630$ \\
\hline & ISI (Dubai, UAE & $=0.829$ & РИНЦ (Russia) & $=0.156$ & PIF (India) & $=1.940$ \\
\hline & GIF (Australia) & $=0.564$ & ESJI $(\mathrm{KZ})$ & $=8.716$ & IBI (India) & $=4.260$ \\
\hline & JIF & $=1.500$ & SJIF (Morocco) & $=5.667$ & OAJI (USA) & $=0.350$ \\
\hline
\end{tabular}

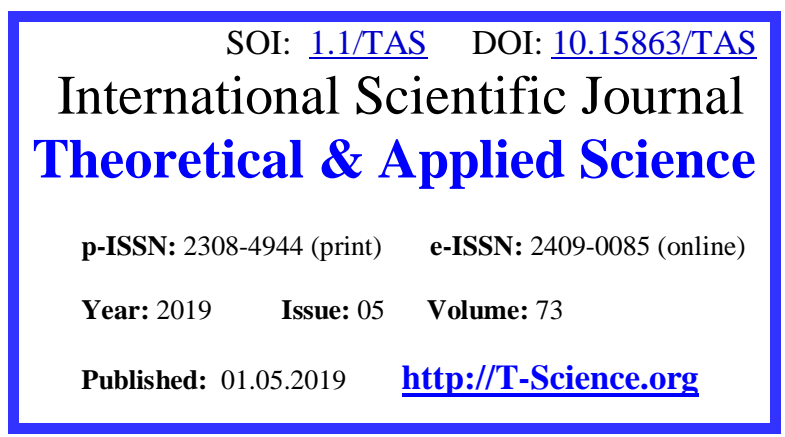

SECTION 1. Theoretical research in mathematics
QR - Issue

QR - Article

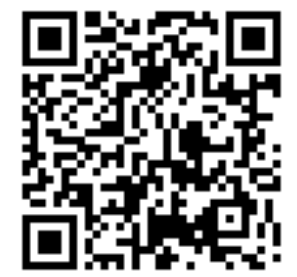

Ablakul Abdirashidov Corresponding member of International Academy, Doctor of Physical and Mathematical Sciences, Docent to department of theoretical and applied mechanics, Samarkand State University, Uzbekistan abdira@mail.ru

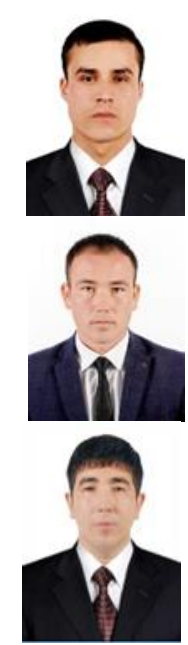

Bekzod Ortikov

Student of Mechanical and Mathematical Faculty, Samarkand State University, Uzbekistan

Nurshod Kadirov Student of Mechanical and Mathematical Faculty, Samarkand State University, Uzbekistan

Akmaljon Abdurashidov Researcher Samarkand State University, Uzbekistan

\title{
APPLICATION OF ADOMIAN DECOMPOSITION METHOD, TAYLOR SERIES METHOD AND A VARIATIONAL ITERATIONS METHOD TO SOLVING A SECOND ORDER ORDINARY DIFFERENTIAL EQUATIONS
}

Abstract: In this paper, the Cauchy problem with the second order ordinary differential equations is solved analytically using the Adomian decomposition method, Taylor series method and the variational iteration method. It is shown that these methods are the most effective and convenient for solving some evolution equations. The obtained approximate solutions were compared, the results of these methods are the same; while the method of decomposition of Adomian can be much simpler, more convenient and more efficient to approach such problems as compared to the method of variational iteration and other traditional methods.

Key words: second order ordinary differential equations, Cauchy problem, variational iteration method, Adomian decomposition method, Taylor series method, exact solutions.

Language: English

Citation: Abdirashidov, A., Ortikov, B., Kadirov, N., \& Abdurashidov, A. (2019). Application of Adomian decomposition method, Taylor series method and a variational iterations method to solving a second order ordinary differential equations. ISJ Theoretical \& Applied Science, 05 (73), 1-5.

Soi: http://s-o-i.org/1.1/TAS-05-73-1 Doi: crossef https://dx.doi.org/10.15863/TAS.2019.05.73.1

\section{Introduction.}

Nonlinear phenomena are of fundamental importance in various fields of science and technology. Nonlinear models of real-world problems are still difficult to solve either numerically or theoretically. Recently, much attention has been paid to the search for better and more efficient approximate or exact, analytical or numerical methods for solving for nonlinear models $[6,7,10,11]$. There are many standard semi-analytical methods for solving linear and nonlinear partial or ordinary differential equations, for example, the Adomian decomposition 


\begin{tabular}{|c|c|c|c|c|c|c|}
\hline \multirow{4}{*}{ Impact Factor: } & ISRA (India) & $=3.117$ & SIS (USA) & $=0.912$ & ICV (Poland) & $=6.630$ \\
\hline & ISI (Dubai, UAI & $=0.829$ & РИНЦ (Russia & $=0.156$ & PIF (India) & $=1.940$ \\
\hline & GIF (Australia) & $=0.564$ & ESJI (KZ) & $=8.716$ & IBI (India) & $=4.260$ \\
\hline & JIF & $=1.500$ & SJIF (Morocce & $=5.667$ & OAJI (USA) & $=0.350$ \\
\hline
\end{tabular}

method, Taylor series method and the variational iterations method [6-12].

The Adomian decomposition method and the variational iterations method is one of the wellknown methods for solving various linear and nonlinear evolution equations. Many studies have proven that these methods are reliable and effective for a wide range of scientific applications, linear and nonlinear equations with bounded and unbounded domains [17]. These methods have no special requirements, such as linearization, small parameters, and so on for nonlinear operators. Below, the Cauchy problem with the second order ordinary differential equations are solved analytically using the Adomian decomposition method, Taylor series method and variational iterations method.

\section{Analysis of the methods.}

1) Adomian decomposition method.

Considering the differential equation below in an operator form as

$$
L u+R u=f
$$

In this case $L$ is mostly the lower order derivative assumed to be invertible, $R$ is other differential operator while $f$ is the source term. Applying $L^{-1}$ to both sides of equation (1) and imposing the given conditions, we have

$$
u=h-L^{-1}(R u)
$$

where the function $h$ represents given conditions and the source term. The standart Adomian decomposition method gives the solution of $u$ by and infinite series of components written as

$$
u=\sum_{n=0}^{\infty} u_{n}
$$

where the components $u_{0}, u_{1}, u_{2}, \ldots$ are determined recursively. Substituting (3) into (2) yields

$$
\sum_{n=0}^{\infty} u_{n}=h-L^{-1}\left[R\left(\sum_{n=0}^{\infty} u_{n}\right)\right]
$$

We then determine the solution by identifying the zeroth components as

$$
u_{0}=h
$$

and the remaining components are written as the recursive relation

$$
u_{n+1}=-L^{-1}\left[R\left(u_{n}\right)\right], \quad n \geq 0
$$

2) Variational iterations method.

Considering the differential equation below in an operator form as

$$
L u+N u=f(t)
$$

where $L$ is linear operator, $N$ is a nonlinear operator and $f(t)$ is known analytical function.

According to the variational iterations method, we can construct a correction functional as follows:

$$
u_{n+1}(t)=u_{n}(t)+\int_{0}^{t} \lambda(\xi)\left[L u_{n}(\xi)+N \tilde{u}_{n}(\xi)-f(\xi)\right] d \xi
$$

where $\lambda$ is a general Lagrange multiplier, which can be identified optimally via the variational theory (He, 2007), the subscript $n$ denotes the $n$th approximation and $\tilde{u}_{n}$ is considered as a restricted variation, i.e., $\delta \tilde{u}_{n}=0$

It is obvious now that the main steps of the variational iterations method require first the determination of the Lagrangian multiplier $\lambda$ that will be identified optimally. Having determined the Lagrangian multiplier, the successive approximations $u_{n+1}, n \geq 0$, of the solution $u$ will be readily obtained upon using any selective function $u_{0}$. Consequently, the solution

$$
u=\lim _{n \rightarrow \infty} u_{n}
$$

Lagrange multiplier can be easily identified as:

$$
\lambda(\xi)=(-1)^{m} \frac{1}{(m-1) !}(\xi-t)^{m-1}
$$

III. Application methods and results.

Example. Consider the second order ordinary differential equations

$$
y^{\prime \prime}-5 y^{\prime}=5, \quad y(0)=1, \quad y^{\prime}(0)=1, \quad 0<x<3 \text {. }
$$

We find the exact solution to the problem

$$
y(x)=0,4 e^{5 x}+0,6-x \text {. }
$$

1) Adomian decomposition method.

$$
y^{\prime \prime}=5+5 y^{\prime} \Rightarrow L^{-1} y^{\prime \prime}=L^{-1}\left(5+5 y^{\prime}\right) \text {, }
$$

where $L(\cdot)=\frac{d^{2}(\cdot)}{d x^{2}}$ and $L^{-1}(\cdot)=\int_{0}^{x} \int_{0}^{x}(\cdot) d \xi$, then

$$
y(x)=1+x+\frac{5 x^{2}}{2}+5 \int_{0}^{x} \int_{0}^{x} y^{\prime}(\xi) d \xi
$$

According to ADM we search for the solution as follows: $y(x)=\sum_{k=0}^{\infty} y_{k}(x)$

This,

$$
\begin{aligned}
& y_{0}+y_{1}+y_{2}+\ldots=1+x+\frac{5 x^{2}}{2}+ \\
& +5 \int_{0}^{x} \int_{0}^{x}\left[y_{0}^{\prime}+y_{1}^{\prime}+y_{2}^{\prime}+\ldots\right] d \xi
\end{aligned}
$$

now

$$
y_{0}=1+x+\frac{5 x^{2}}{2}
$$




\begin{tabular}{|c|c|c|c|c|c|c|}
\hline \multirow{4}{*}{ Impact Factor: } & ISRA (India) & $=3.117$ & SIS (USA) & $=0.912$ & ICV (Poland) & $=6.630$ \\
\hline & ISI (Dubai, UAE & $=0.829$ & РИНЦ (Russia) & $=0.156$ & PIF (India) & $=1.940$ \\
\hline & GIF (Australia) & $=0.564$ & ESJI (KZ) & $=8.716$ & IBI (India) & $=4.260$ \\
\hline & JIF & $=1.500$ & SJIF (Morocco & $=5.667$ & OAJI (USA) & $=0.350$ \\
\hline
\end{tabular}

$$
\begin{gathered}
y_{1}=5 \int_{0}^{x} \int_{0}^{x} y_{0}^{\prime} d \xi=\frac{5 x^{2}}{2}+\frac{25 x^{3}}{3 !} \\
y_{2}=5 \int_{0}^{x} \int_{0}^{x} y_{1}^{\prime} d \xi=\frac{25 x^{3}}{3 !}+\frac{125 x^{4}}{4 !} \\
y_{n}=5 \int_{0}^{x} \int_{0}^{x} y_{n-1}^{\prime} d \xi=\frac{5^{n} x^{n+1}}{(n+1) !}+\frac{5^{n+1} x^{n+2}}{(n+2) !} \quad \text { and so on. } \\
y(x)=\sum_{k=0}^{\infty} y_{k}(x)=1+x+\sum_{n=1}^{\infty} \frac{2 \cdot 5^{n} x^{n+1}}{(n+1) !}= \\
=1-x+\sum_{n=0}^{\infty} \frac{2 \cdot 5^{n} x^{n+1}}{(n+1) !}=1-x++0,4 \cdot \sum_{n=0}^{\infty} \frac{(5 x)^{n+1}}{(n+1) !} \\
=0,6-x+0,4 \cdot \sum_{n=0}^{\infty} \frac{(5 x)^{n}}{n !}=0,6-x+0,4 \cdot e^{5 x} .
\end{gathered}
$$

The found solution to be compatible with the exact solution.

2) Variational iterations method. To solve the VIM problem, we first use the replacement

$$
y(x)=1+\int_{0}^{x} z(\xi) d \xi, \quad z(0)=1 .
$$

The formula of VIM is

$$
z_{k+1}(x)=z_{k}(x)+\int_{0}^{x} \lambda(\xi)\left(z_{k}^{\prime}-5 z_{k}-5\right) d \xi
$$

Where $\lambda(\xi)$ - Lagrange multiplier, and for the stationary case $\left.\lambda^{\prime}(\xi)\right|_{\xi=x}=0 ; 1+\left.\lambda(\xi)\right|_{\xi=x}=0$ and from here we have $\lambda(\xi)=-1$. Then we have

$$
z_{k+1}(x)=z_{k}(x)-\int_{0}^{x}\left(z_{k}^{\prime}-5 z_{k}-5\right) d \xi .
$$

Now applying VIM, we get the following results: $z_{0}=1 ; \quad z_{1}=1+10 x ; \quad z_{2}=1+10 x+25 x^{2} ;$ $z_{3}=1+10 x+25 x^{2}+\frac{125 x^{3}}{3}$;

$$
\begin{aligned}
& z_{4}=1+10 x+25 x^{2}+\frac{125 x^{3}}{3}+\frac{625 x^{4}}{12} ; \ldots ; \\
& z_{n}=2 \cdot \sum_{k=0}^{n} \frac{(5 x)^{n}}{n !}-1 \quad \text { and so on. }
\end{aligned}
$$

According to them

$$
\begin{gathered}
y_{0}=1+x ; \\
y_{1}=x+5 x^{2} ; \\
y_{2}=x+5 x^{2}+\frac{25 x^{3}}{3} ; \\
y_{3}=x+5 x^{2}+\frac{25 x^{2}}{3}+\frac{125 x^{4}}{12} ; \\
y_{4}=x+5 x^{2}+\frac{25 x^{2}}{3}+\frac{125 x^{4}}{12}+\frac{625 x^{5}}{120} ; \ldots ; \\
y_{n}=1-x+0,4 \cdot \sum_{k=0}^{n} \frac{(5 x)^{n+1}}{(n+1) !} \text { and so on. }
\end{gathered}
$$

3) Taylor series method.

$$
\begin{gathered}
y^{\prime \prime}=5+5 y^{\prime}, \quad y(0)=1, y^{\prime}(0)=1, \\
y^{(n)}=\left.5 y^{(n-1)} \Rightarrow \quad y^{(n)}\right|_{x=0}=\left.5 y^{(n-1)}\right|_{x=0}, \quad n>2 \\
y^{\prime \prime}(0)=10 ; \quad y^{\prime \prime \prime}(0)=50 ; \quad y^{I V}(0)=250 ; \\
y^{V}(0)=1250 ; \ldots ; \quad y^{(n)}(0)=2 \cdot 5^{n-1} \quad \text { and so on. }
\end{gathered}
$$
given by

Recall that the Taylor expansion of $y(x)$ is

$$
\begin{aligned}
& y(x)=y(0)+\frac{y^{\prime}(0)}{1 !} x+\frac{y^{\prime \prime}(0)}{2 !} x^{2}+ \\
& +\frac{y^{\prime \prime \prime}(0)}{3 !} x^{3}+\ldots+\frac{y^{(n)}(0)}{n !} x^{n}+\ldots
\end{aligned}
$$

then

$$
\begin{aligned}
& y(x)=1+x+5 x^{2}+\frac{50}{3 !} x^{3}+\ldots+\frac{2 \cdot 5^{n-1}}{n !} x^{n}+\ldots= \\
& =0,6-x+0,4 e^{5 x}
\end{aligned}
$$

The result of the difference between the exact and approximate solution alongside the Absolute error $E_{A}$, shown in the Table I below:

Table I.

\begin{tabular}{|l|l|l|l|l|l|l|l|}
\hline \multirow{2}{*}{$X$} & Exact & Approximate solutions $y_{n}(x)$ & \multicolumn{5}{l|}{ Absolute error $E_{A}$} \\
\cline { 3 - 8 } & solution $y(x)$ & $n=20$ & $n=25$ & $n=30$ & $n=20$ & $n=25$ & $n=30$ \\
\hline 0 & 1 & 1 & 1 & 1 & 0 & 0 & 0 \\
\hline 0,2 & 1,487312731 & 1,48731273 & 1,48731273 & 1,48731273 & $2,22045 \mathrm{E}-16$ & $2,22045 \mathrm{E}-16$ & $2,22045 \mathrm{E}-16$ \\
\hline 0,4 & 3,15562244 & 3,15562244 & 3,15562244 & 3,15562244 & $1,86517 \mathrm{E}-14$ & $8,88178 \mathrm{E}-16$ & $8,88178 \mathrm{E}-16$ \\
\hline 0,6 & 8,034214769 & $\begin{array}{l}8,03421476 \\
9\end{array}$ & $\begin{array}{l}8,03421476 \\
9\end{array}$ & $\begin{array}{l}8,03421476 \\
9\end{array}$ & $9,47278 \mathrm{E}-11$ & $3,55271 \mathrm{E}-15$ & 0 \\
\hline
\end{tabular}




\begin{tabular}{|c|c|c|c|c|c|c|}
\hline \multirow{4}{*}{ Impact Factor: } & ISRA (India) & $=3.117$ & SIS (USA) & $=0.912$ & ICV (Poland) & $=6.630$ \\
\hline & ISI (Dubai, UAE & $=0.829$ & РИНЦ (Russia & $=0.156$ & PIF (India) & $=1.940$ \\
\hline & GIF (Australia) & $=0.564$ & ESJI (KZ) & $=8.716$ & IBI (India) & $=4.260$ \\
\hline & JIF & $=1.500$ & SJIF (Morocce & $=5.667$ & OAJI (USA) & $=0.350$ \\
\hline
\end{tabular}

\begin{tabular}{|c|c|c|c|c|c|c|c|}
\hline 0,8 & 21,63926001 & $\begin{array}{l}21,6392599 \\
7\end{array}$ & $\begin{array}{l}21,6392600 \\
1\end{array}$ & $\begin{array}{l}21,6392600 \\
1\end{array}$ & 4,19982E-08 & $5,22959 \mathrm{E}-12$ & $1,06581 \mathrm{E}-14$ \\
\hline 1 & 58,96526364 & $\begin{array}{l}58,9652588 \\
3\end{array}$ & $\begin{array}{l}58,9652636 \\
4\end{array}$ & $\begin{array}{l}58,9652636 \\
4\end{array}$ & $4,81408 \mathrm{E}-06$ & 1,81063E-09 & $2,77112 \mathrm{E}-13$ \\
\hline 1,2 & 160,7715174 & $\begin{array}{l}160,771282 \\
6\end{array}$ & $\begin{array}{l}160,771517 \\
2\end{array}$ & $\begin{array}{l}160,771517 \\
4\end{array}$ & $\begin{array}{l}0,00023481 \\
3\end{array}$ & 2,16923E-07 & $7,92966 \mathrm{E}-11$ \\
\hline 1,4 & 437,8532634 & $\begin{array}{l}437,846904 \\
9\end{array}$ & $\begin{array}{l}437,853250 \\
9\end{array}$ & $\begin{array}{l}437,853263 \\
4\end{array}$ & $\begin{array}{l}0,00635842 \\
9\end{array}$ & $1,25177 \mathrm{E}-05$ & $9,80179 \mathrm{E}-09$ \\
\hline 1,6 & 1191,383195 & $\begin{array}{l}1191,27114 \\
9\end{array}$ & $\begin{array}{l}1191,38277 \\
1\end{array}$ & $\begin{array}{l}1191,38319 \\
4\end{array}$ & $\begin{array}{l}0,11204574 \\
9\end{array}$ & $\begin{array}{l}0,00042346 \\
9\end{array}$ & $6,40278 \mathrm{E}-07$ \\
\hline 1,8 & 3240,033571 & $\begin{array}{l}3238,60985 \\
3\end{array}$ & $\begin{array}{l}3240,02403 \\
7\end{array}$ & $\begin{array}{l}3240,03354 \\
5\end{array}$ & $\begin{array}{l}1,42371786 \\
7\end{array}$ & $\begin{array}{l}0,00953379 \\
5\end{array}$ & $2,5708 \mathrm{E}-05$ \\
\hline 2 & 8809,186318 & 8795,19281 & $\begin{array}{l}8809,03054 \\
4\end{array}$ & $\begin{array}{l}8809,18561 \\
5\end{array}$ & $\begin{array}{l}13,9935076 \\
6\end{array}$ & $\begin{array}{l}0,15577356 \\
6\end{array}$ & $\begin{array}{l}0,00070341 \\
9\end{array}$ \\
\hline 2,2 & 23948,05669 & 23836,186 & $\begin{array}{l}23946,0916 \\
1\end{array}$ & $\begin{array}{l}23948,0425 \\
7\end{array}$ & $\begin{array}{l}111,870682 \\
7\end{array}$ & 1,96507678 & $\begin{array}{l}0,01411860 \\
1\end{array}$ \\
\hline 2,4 & 65100,11657 & $\begin{array}{l}64345,0816 \\
5\end{array}$ & $\begin{array}{l}65080,0811 \\
2\end{array}$ & $\begin{array}{l}65099,8970 \\
7\end{array}$ & $\begin{array}{l}755,034920 \\
5\end{array}$ & $\begin{array}{l}20,0354488 \\
3\end{array}$ & $\begin{array}{l}0,21950072 \\
9\end{array}$ \\
\hline 2,6 & 176963,3568 & $\begin{array}{l}172537,132 \\
8\end{array}$ & $\begin{array}{l}176792,402 \\
1\end{array}$ & $\begin{array}{l}176960,601 \\
9\end{array}$ & $\begin{array}{l}4426,22398 \\
9\end{array}$ & $\begin{array}{l}170,954681 \\
3\end{array}$ & $\begin{array}{l}2,75495219 \\
2\end{array}$ \\
\hline 2,8 & 481039,5137 & $\begin{array}{l}457993,570 \\
3\end{array}$ & $\begin{array}{l}479785,155 \\
9\end{array}$ & $\begin{array}{l}481010,685 \\
9\end{array}$ & $\begin{array}{l}23045,9433 \\
7\end{array}$ & 1254,35775 & $\begin{array}{l}28,8277669 \\
5\end{array}$ \\
\hline 3 & 1307604,549 & 1199111,21 & $\begin{array}{l}1299517,12 \\
6\end{array}$ & $\begin{array}{l}1307346,54 \\
1\end{array}$ & $\begin{array}{l}108493,338 \\
5\end{array}$ & $\begin{array}{l}8087,42320 \\
2\end{array}$ & $\begin{array}{l}258,008045 \\
7\end{array}$ \\
\hline
\end{tabular}

Since the exact solution function is grown, also is absolute error high, so we also investigate relative errors $E_{R}$, shown in the Table II below:

Table II.

\begin{tabular}{|l|l|l|l|}
\hline \multirow{2}{*}{$x$} & \multicolumn{3}{|c|}{ Relative error $E_{R}$} \\
\cline { 2 - 4 } & \multicolumn{1}{|c|}{$n=20$} & \multicolumn{1}{|c|}{$n=25$} & \multicolumn{1}{c|}{$n=30$} \\
\hline 0 & 0 & 0 & 0 \\
\hline 0,2 & $1,49292 \mathrm{E}-16$ & $1,49292 \mathrm{E}-16$ & $1,49292 \mathrm{E}-16$ \\
\hline 0,4 & $5,91064 \mathrm{E}-15$ & $2,81459 \mathrm{E}-16$ & $2,81459 \mathrm{E}-16$ \\
\hline 0,6 & $1,17905 \mathrm{E}-11$ & $4,42198 \mathrm{E}-16$ & 0 \\
\hline 0,8 & $1,94083 \mathrm{E}-09$ & $2,41672 \mathrm{E}-13$ & $4,92537 \mathrm{E}-16$ \\
\hline 1 & $8,16426 \mathrm{E}-08$ & $3,07067 \mathrm{E}-11$ & $4,69957 \mathrm{E}-15$ \\
\hline 1,2 & $1,46054 \mathrm{E}-06$ & $1,34927 \mathrm{E}-09$ & $4,93225 \mathrm{E}-13$ \\
\hline 1,4 & $1,45218 \mathrm{E}-05$ & $2,85889 \mathrm{E}-08$ & $2,2386 \mathrm{E}-11$ \\
\hline 1,6 & $9,40468 \mathrm{E}-05$ & $3,55443 \mathrm{E}-07$ & $5,37424 \mathrm{E}-10$ \\
\hline 1,8 & 0,000439415 & $2,9425 \mathrm{E}-06$ & $7,93449 \mathrm{E}-09$ \\
\hline 2 & 0,001588513 & $1,76831 \mathrm{E}-05$ & $7,98506 \mathrm{E}-08$ \\
\hline 2,2 & 0,004671389 & $8,20558 \mathrm{E}-05$ & $5,89551 \mathrm{E}-07$ \\
\hline 2,4 & 0,011598058 & 0,000307764 & $3,37174 \mathrm{E}-06$ \\
\hline 2,6 & 0,025012093 & 0,000966046 & $1,55679 \mathrm{E}-05$ \\
\hline 2,8 & 0,047908629 & 0,002607598 & $5,99281 \mathrm{E}-05$ \\
\hline 3 & 0,082971062 & 0,006184915 & 0,000197314 \\
\hline
\end{tabular}

\section{Conclusions.}

In this work, ADM, TSM and VIM were successfully applied to solve the Cauchy problem with the second order ordinary differential equations. It is obvious that VIM an ADM are very powerful and effective methods for finding analytical solutions for wide classes of problems. It is worth noting that these two methods are a quick convergence of solutions. Application of ADM to the problems discussed has more advantages than VIM and most other methods; it overcomes the difficulties in calculating other 


\begin{tabular}{|c|c|c|c|c|c|c|}
\hline \multirow{4}{*}{ Impact Factor: } & ISRA (India) & $=3.117$ & SIS (USA) & $=0.912$ & ICV (Poland) & $=6.630$ \\
\hline & ISI (Dubai, UAI & $=0.829$ & РИНЦ (Russia & $=0.156$ & PIF (India) & $=1.940$ \\
\hline & GIF (Australia) & $=0.564$ & ESJI (KZ) & $=8.716$ & IBI (India) & $=4.260$ \\
\hline & JIF & $=1.500$ & SJIF (Morocco & $=5.667$ & OAJI (USA) & $=0.350$ \\
\hline
\end{tabular}

methods and auxiliary parameters; it helps us to obtain a solution for smaller approximations. Also, the ADM does not require changing some parameters in the equation, therefore, the calculations are simple and straightforward.

\section{References:}

1. Abdirashidov, A., Kadirov, N. X., Ortikov, B. B., \& Abdurashidov, A. A. (2018). Exact solution of fractional diffusion equations using the variational iteration method and Adomian decomposition method. International Scientific Journal «Theoretical \& Applied Science», №5, pp.101-107.

2. Abdurashidov, A. A., Ortiqov, B. B., Qadirov, N. X., \& Abdirashidov, A. (2018). Exact solution of nonlinear equations Burgers-Huxley, Korteweg-de Vries-Burgers and Klein-Gordon using the modified simple equation method. International Scientific Journal «Theoretical \& Applied Science», №3, pp.101-107.

3. Abdirashidov, A., Karshiyev, A., Ortikov, B. B., \& Kadirov, N. X. (2018). Application of approximate Adomian decomposition method and a variational iterations method to solving a Cauchy problem with the heat dissipation and Laplace equations. International Scientific Journal «Theoretical \& Applied Science», №12, pp.323-329.

4. Abdurashidov, A., Ortiqov, B. B., Qadirov, N. X., \& Abdirashidov, A. A. (2018). Solution of fractional telegraph and diffusion equations using the approximation methods. International Scientific Journal «Theoretical \& Applied Science», №10, pp.30-36.
5. Adomian, G. (1994). Solving Frontier Problems of Physics: The Decomposition Method. Boston, MA: Kluwer.

6. Wazwaz, A. M. (2011). Linear and Nonlinear Integral Equations. (p.658). Higher Education Press, Berlin Heidelberg.

7. Wazwaz, A. M. (2009). Partial Differential Equations and Solitary Waves Theory. (p.761). Higher Education Press, Berlin Heidelberg.

8. Filippov, A. F. (2000). Sbornik zadach po differensialnbım uravneniyam. (p.176). Ijevsk, NIS.

9. Azlarov, T. A., \& Mansurov, X. (1989). Matematicheskiy analiz. II chast. (p.424). Tashkent: "O'qituvchi”.

10. Bisadze, A. V., \& Kalinichenko, D. F. (1985). Sbornik zadach po uravneniyam matematicheskoy fiziki. Uchebnoye posobiye. 2-ye izd., dop. (p.310). Moscow: Nauka.

11. Ibragimov, N. X. (2007). Prakticheskiy kurs differensialnblx uravneniy i matematicheskogo modelirovaniya. (p.421). Nijniy Novgorod: Izdvo Nijegorodskogo gosuniversiteta.

12. Kudryashov, N. A. (2010) Metods nelineynoy matematicheskoy fiziki: Uchebnoye posobiye. 2ye izd. (p.368). Dolgoprudniy: Intellekt. 